\title{
EMBEDDINGS PROPERTIES ON HERZ-TYPE BESOV AND TRIEBEL-LIZORKIN SPACES
}

\author{
DOUADI DRIHEM
}

Abstract. We study the embeddings problems on Herz-type Besov-Triebel-Lizorkin spaces. In particular we will give a proof of the Sobolev-type embedding for these function spaces. All these results generalize the classical results on Besov and Triebel-Lizorkin spaces.

Mathematics subject classification (2010): 46E35.

Keywords and phrases: Besov spaces, Triebel-Lizorkin spaces, Herz spaces, embedding.

\section{REFERENCES}

[1] A. BAernstein II And E. T. SAWyer, Embedding and multiplier theorems for $H^{p}\left(\mathbb{R}^{n}\right)$, Mem. Amer. Math. Soc. 53, 318 (1985).

[2] C. HERZ, Lipschitz spaces and Bernstein's theorem on absolutely convergent Fourier transforms, J. Math. Mech. 18 (1968), 283-324.

[3] H. BReZis AND P. Mironescu, Gagliardo-Nirenberg composition and products in fractional Sobolev spaces, J. Evol. Equ. 1 (2001), 387-404.

[4] L. DiENING, P. HÄSTÖ AND S. RoudENKo, Function spaces of variable smoothness and integrability, J. Funct. Anal. 256, 6 (2009), 1731-1768.

[5] H. G. Feichtinger And F. Weisz, Herz spaces and summability of Fourier transforms, Math. Nachr. 281, 3 (2008), 309-324.

[6] E. HeRnANDEZ, G. WeisS AND D. YANG, The $\varphi$-transform and wavelet characterizations of Herztype spaces, Collect. Math. 47, 3 (1996), 285-320.

[7] E. HernandeZ And D. YAnG, Interpolation of Herz-type Hardy spaces and applications, Math. Nachr. 42 (1998), 564-581.

[8] J. JOHNSEN AND W. SICKEL, A direct proof of Sobolev embeddings for quasi-homogeneous LizorkinTriebel spaces with mixed norms, J. Funct. Spaces Appl. 5 (2007), 183-198.

[9] H. Kozono AND M. YAmaZAKI, Semilinear heat equations and the Navier-Stokes equation with distributions in new function spaces as initial data, Comm. Partial Differential Equations 19, 5-6 (1994), 959-1014.

[10] A. L. MazzUCATo, Besov-Morrey spaces: function space theory and applications to nonlinear PDE, Trans. Amer. Math. Soc. 355 (2003), 1297-1364.

[11] A. Mazzucato, Decomposition of Besov-Morrey spaces in Harmonic Analysis at Mount Holyoke, AMS Series in Contemporary Mathematics. 320 (2003), 279-294.

[12] S. LU AND D. YANG, The local versions of $H^{p}\left(\mathbb{R}^{n}\right)$ spaces at the origin, Studia Math. 116 (1995), $103-131$.

[13] S. LU AND D. YANG, The decomposition of the weighted Herz spaces on $\mathbb{R}^{n}$ and its applications, Sci. in China (Ser.A) 38 (1995), 147-158.

[14] S. Lu And D. YAng, Herz-type Sobolev and Bessel potential spaces and their applications, Sci. in China (Ser.A) 40 (1997), 113-129.

[15] Y. Sawano And H. Tanaka, Decompositions of Besov-Morrey spaces and Triebel-LizorkinMorrey spaces, Math. Z. 257 (2007), 871-905.

[16] M. A. RAGUS A, Homogeneous Herz spaces and regularity results, Nonlinear Anal. 71 (2009), e1909e1914.

[17] H. TRIEBEL, Theory of function spaces, Birkhäuser, Basel, 1983. 
[18] H. TRIEBEL, Theory of function spaces II, Birkhä user, Basel, 1992.

[19] H. TRIEBEL, Theory of function spaces III, Birkhä user, Basel, 2006.

[20] H. WANG, Decomposition for Morrey type Besov-Triebel spaces, Math. Nachr. 282, 5 (2009), 774 787.

[21] J. XU And D. YAng, Applications of Herz-type Triebel-Lizorkin spaces, Acta. Math. Sci. (Ser. B) 23 (2003), 328-338.

[22] J. Xu And D. YAng, Herz-type Triebel-Lizorkin spaces I, Acta Math. Sci. (English Ed.) 213 (2005), 643-654.

[23] J. Xu, Some properties on Herz-type Besov spaces (in chinese), J. Hunan Univ. (Natural Sci.) 30, 5 (2003), 75-78.

[24] J. Xu, Pointwise multipliers of Herz-type Besov spaces and their applications, Appl. Math. 17, 1 (2004), 115-121.

[25] J. XU, Equivalent norms of Herz-type Besov and Triebel-Lizorkin spaces, J. Funct. Spaces Appl. 3 (2005), 17-31. 